\title{
Mixed ductal-acinar cell carcinoma of the pancreas: A case report
}

\author{
TAKAHIRO SHIGAKI $^{1}$, TORU HISAKA ${ }^{1}$, FUMIHIKO FUJITA ${ }^{1}$, HIRONORI KUSANO ${ }^{2}$, YOSHIKI NAITO ${ }^{3}$, \\ RYUTA MIDORIKAWA ${ }^{1}$, TAKAFUMI OHCHI ${ }^{1}$, ICHITARO SHIRATSUCHI ${ }^{1}$, \\ ATSUHIRO HIDAKA ${ }^{4}$, TAKAHO TANAKA ${ }^{4}$ and YOSHITO AKAGI $^{1}$ \\ ${ }^{1}$ Department of Surgery, Kurume University Hospital; ${ }^{2}$ Department of Pathology, Kurume University School of Medicine; \\ ${ }^{3}$ Department of Diagnostic Pathology, Kurume University Hospital, Kurume, Fukuoka 830-0011; \\ ${ }^{4}$ Department of Surgery, Tagawa Hospital, Tagawa, Fukuoka 826-8585, Japan
}

Received August 7, 2018; Accepted February 25, 2019

DOI: $10.3892 /$ mco.2019.1831

\begin{abstract}
Mixed carcinoma of the pancreas is defined as the concurrent existence of pancreatic ductal carcinoma, acinar cell carcinoma, and/or islet cell carcinoma within the same neoplasm. We herein report a rare case of mixed ductal-acinar cell carcinoma in a 74-year-old man who was undergoing treatment for hypertension and diabetes at another hospital. After an abrupt worsening of his blood glucose control, the patient was referred to our hospital for further evaluation. Abdominal contrast-enhanced computed tomography and magnetic resonance imaging revealed a tumor with a multilocular cystic lesion in the head of the pancreas. Endoscopic retrograde cholangiopancreatography revealed obstruction of the main pancreatic duct and dilation of the dorsal pancreatic duct; in addition, adenocarcinoma was detected in the pancreatic juice cytology. Based on the abovementioned findings, the patient was diagnosed with carcinoma of the pancreatic head and underwent subtotal stomach-preserving pancreaticoduodenectomy. Based on the histopathological and immunohistochemical findings, the patient was diagnosed with mixed ductal-acinar cell carcinoma. The patient was prescribed TS-1 as postoperative adjuvant chemotherapy upon discharge. However, treatment was discontinued 2 months later due to marked general malaise, and the patient succumbed to tumor recurrence in the residual pancreas 12 months after the surgery.
\end{abstract}

Correspondence to: Dr Takahiro Shigaki, Department of Surgery, Kurume University Hospital, 67 Asahi-machi, Kurume, Fukuoka 830-0011, Japan

E-mail: shigakitakahiro@gmail.com

Abbreviations: CT, computed tomography; IPMN, intraductal papillary mucinous neoplasm; ITPN, intraductal tubulopapillary neoplasm

Key words: combined tumor, pancreatic ductal carcinoma, acinar cell carcinoma, exocrine tumor, immunohistochemistry, TS-1

\section{Introduction}

Mixed carcinoma of the pancreas is defined as the coexistence of exocrine and endocrine carcinomatous components within the same pancreatic neoplasm. Broadly stated, mixed carcinoma of the pancreas involves the coexistence of ductal and acinar cell carcinoma (1). Acinar cell carcinoma accounts for no more than $1-2 \%$ of all adult pancreatic tumors (2). The World Health Organization (WHO) classification categorizes mixed ductal-acinar cell carcinoma as a sub-class of acinar cell neoplasms (3), and its diagnosis is established when 25\% of the tumor displays acinar and ductal elements based on the pathological findings.

Mixed ductal-acinar cell carcinoma is extremely rare, with only 21 cases reported in the English and Japanese literature to date (4-10), and its clinicopathological characteristics have not been clearly determined thus far. The current study herein describes our experience with a case of mixed ductal-acinar cell carcinoma in a 74-year-old male patient and discuss the relevant literature.

\section{Case report}

A 74-year-old man presented with an abrupt deterioration of blood glucose control, while undergoing treatment for diabetes and hypertension by a local physician in February 2016. Oral medication for both diseases had been prescribed at the age of 71 years. The patient had a history of pharyngeal cancer, for which he had received surgical treatment at the age of 69 years. The family history was unremarkable. Ultrasonography and computed tomography (CT) examination revealed a tumor measuring $30 \mathrm{~mm}$ in the head of the pancreas, as well as dilation of the main pancreatic duct. The patient was then referred to Tagawa Hospital for extensive evaluation and treatment. On physical examination, the patient's height and body weight were $167.0 \mathrm{~cm}$ and $52.5 \mathrm{~kg}$, respectively. No anemia was detected in the palpebral conjunctiva, and the patient did not have jaundice, abdominal tenderness, or back pain. No other abnormal findings were observed. The results of the blood tests revealed that tumor marker levels were high. Carcinoembryonic antigen, $6.2 \mathrm{ng} / \mathrm{ml}$ (normal range, $<5.0 \mathrm{ng} / \mathrm{ml}$ ); carbohydrate antigen $19-9,1131.4 \mathrm{U} / \mathrm{ml}$ (normal range, $<37.0 \mathrm{U} / \mathrm{ml}$ ); DUPAN-2, $240 \mathrm{U} / \mathrm{ml}$ (normal range, $<150 \mathrm{U} / \mathrm{ml}$ ) and SPan-1, $140.1 \mathrm{U} / \mathrm{ml}$ 
(normal range, $<30.0 \mathrm{U} / \mathrm{ml}$ ). Abnormal blood glucose levels were also observed [hemoglobin A1c, 9.0\% (normal range, 4.3-5.8\%); fasting blood sugar, $381 \mathrm{mg} / \mathrm{dl}$ (normal range, 80-109 mg/dl)].

An abdominal ultrasound examination was performed, which revealed a well-differentiated solid tumor, measuring $\sim 35 \times 30 \mathrm{~mm}$, with cystic components. In addition, the main pancreatic duct was dilated peripheral to the tumor. An abdominal contrast-enhanced $\mathrm{CT}$ scan revealed a mixed cystic and solid tumor measuring $\sim 30 \mathrm{~mm}$ in the head of the pancreas, and dilation of the main pancreatic duct in the body and tail of the pancreas. Microcysts were visible in the dorsal pancreas. There was no invasion into the portal vein or the superior mesenteric vein; however, the lower common bile duct and intrapancreatic bile duct were compressed by the tumor. The part of the bile duct cranial to the lower common bile duct and intrapancreatic bile duct was dilated towards the head of the pancreas (Fig. 1A). The surrounding lymph nodes were not enlarged, and there were no findings indicative of distant metastasis or peritoneal dissemination. Magnetic resonance cholangiopancreatography identified a multilocular cystic lesion measuring $40 \mathrm{~mm}$, presenting as an aggregation of microcysts in the head of the pancreas with a solid component. Dilation of the pancreatic duct was observed towards the tail of the pancreas (Fig. 1B).

Endoscopic retrograde cholangiopancreatography revealed stenosis of the lower bile duct $10 \mathrm{~mm}$ peripheral to the papilla of Vater. On pancreatography, almost none of the main pancreatic duct in the head of the pancreas was visualized, and dilation of the pancreatic duct dorsal to the head and body of the pancreas was observed. Concurrently, the pancreatic juice cytology revealed adenocarcinoma (Fig. 2).

The abovementioned findings indicated an intraductal proliferative neoplasm with mixed solid and cystic components. Primary pancreatic cancer, pancreatic cancer with intraductal papillary mucinous neoplasm (IPMN), and pancreatic cancer with intraductal tubulopapillary neoplasm (ITPN) were considered in the differential diagnosis, and subtotal stomach-preserving pancreaticoduodenectomy was performed. During surgery, a tumor was identified in the head of the pancreas, which was firm and elastic in texture, measuring $40 \mathrm{~mm}$ in greatest diameter. The tumor was adjacent to the portal vein and the superior mesenteric vein; however, there was no gross invasion, and the tumor could be easily separated from these structures. Neither liver metastasis nor peritoneal dissemination were observed, and lymph node metastasis was not readily identified intraoperatively. As scheduled, subtotal stomach-preserving pancreaticoduodenectomy was performed, along with reconstruction per Child's method. The surgical specimen included a poorly differentiated tumor with mixed cystic and solid components (Fig. 3). On histological examination, the lesion was primarily located in the main pancreatic duct; clear boundaries and portal invasion were observed (Fig. 4A). The lesion comprised two components (Fig. 4B): One component formed irregularly sized tubular structures, which upon immunohistological examination were positive for epithelial tumor markers and carbohydrate antigen 19-9, therefore suggesting ductal carcinoma (Fig. 4C). The other component exhibited proliferation of tumor cells with oval-shaped nuclei and eosinophilic cytoplasm, forming acinar structures. Upon immunostaining, this component was diffusely positive for trypsin, suggestive of acinar cell carcinoma (Fig. 4D). Due to the concurrent existence of both components in the same tumor, the patient was diagnosed with mixed ductal-acinar carcinoma of the pancreas.

The patient's postoperative progress was uneventful, and no complications were reported. On day 27 , the patient was discharged from the hospital and was prescribed titanium silicate (TS)-1 as postoperative adjuvant chemotherapy; however, due to marked general malaise, TS-1 was discontinued 2 months later. The patient succumbed to recurrence of tumor in the residual pancreas 12 months after the surgery.

\section{Discussion}

Although several cases of mixed acinar-endocrine carcinoma of the pancreas, composed of both acinar and endocrine tumor cells, have been reported, there have only been a few cases of resected mixed ductal-acinar cell carcinomas (1). The World Health Organization (WHO) classification categorizes mixed ductal-acinar cell carcinoma as a sub-class of acinar cell neoplasms (3). The diagnosis of mixed ductal-acinar cell carcinoma is established when $25 \%$ of the tumor displays acinar and ductal elements, based on pathological findings.

Mixed ductal-acinar cell carcinoma is extremely rare and, to the best of our knowledge, only 21 cases have been reported in the English and Japanese literature to date (4-10) (Table I). According to these reports, the mean age of the patients was 69.8 years, the male:female ratio was $15: 6$, and the mean diameter of the tumors was $42.8 \mathrm{~mm}$. In six cases the tumors were present in the tail of the pancreas, while in the remaining cases, the tumors were located in the head of the pancreas.

Preoperative diagnostic imaging of mixed tumors often reflects imaging findings for pancreatic ductal carcinoma and acinar cell carcinoma. On contrast-enhanced CT, pancreatic ductal carcinoma appears as a solid tumor with poor vascularity, whereas acinar cell carcinoma often has relatively rich vascularity and, in case of necrosis, cystic components are often observed. In the present case, we observed a mixed cystic/solid tumor in the head of the pancreas with an acinar cell carcinomatous component in addition to typical pancreatic ductal carcinoma. Histologically, no necrosis was observed, but there was stenosis of the pancreatic duct due to the protrusion of the tumor into the duct lumen; therefore, dilation of the main pancreatic duct distal to the head of the pancreas was observed.

Moreover, the present case exhibited pathological characteristics of both pancreatic ductal carcinoma and acinar cell carcinoma. The acinar cell carcinoma consisted of granular, eosinophilic cells forming acinar structures, while the pancreatic ductal carcinoma consisted of tubular formations. The differential diagnosis of tumors consisting primarily of cystic lesions originating from the pancreatic duct include IPMN and ITPN. There were no papillae observed in the present case, but rather conspicuous tubular structures were observed, which differentiated it from IPMN. The presence of tubular structures resembles ITPN; however, the mucous production in the present case ruled out ITPN. On immunohistochemistry, the acinar cell carcinoma component was diffusely positive for 
A

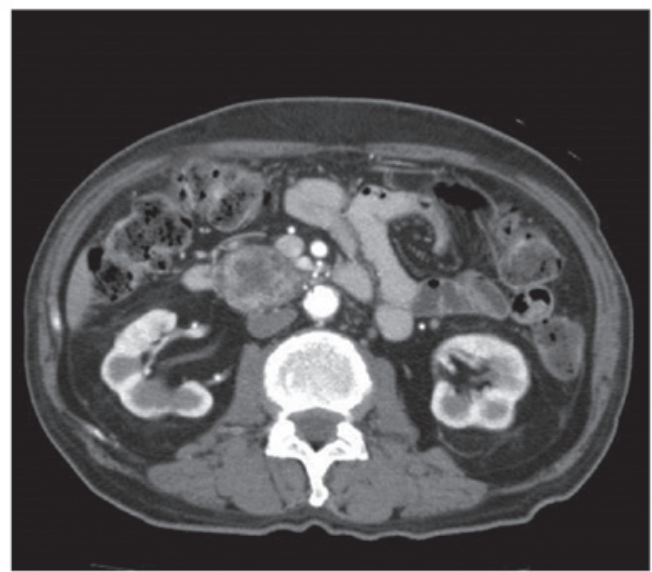

B

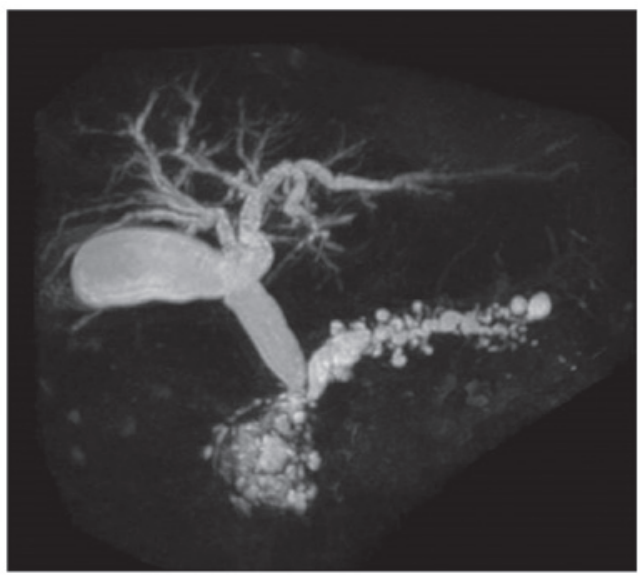

Figure 1. Computed tomography (CT) and magnetic resonance cholangiopancreatography (MRCP) findings. (A) Abdominal contrast-enhanced CT showing dilation of the bile duct portion cranial to the head of the pancreas; (B) MRCP revealed a multilocular cystic lesion in the head of the pancreas; dilation of the pancreatic duct was observed towards the tail of the pancreas

A

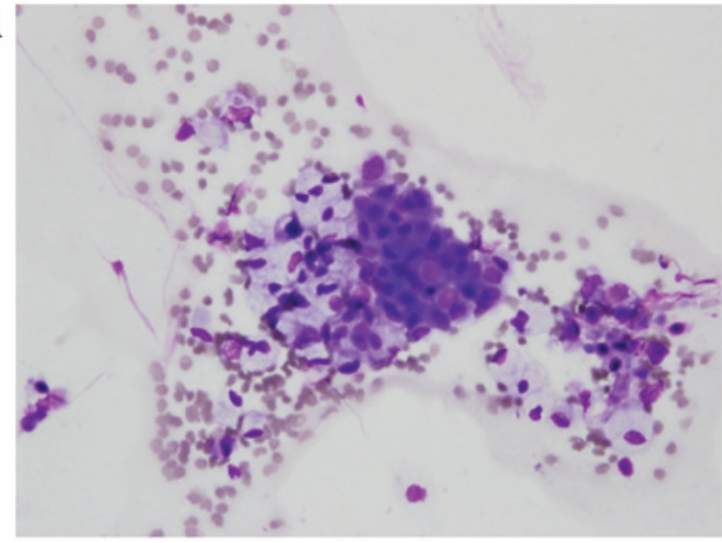

B

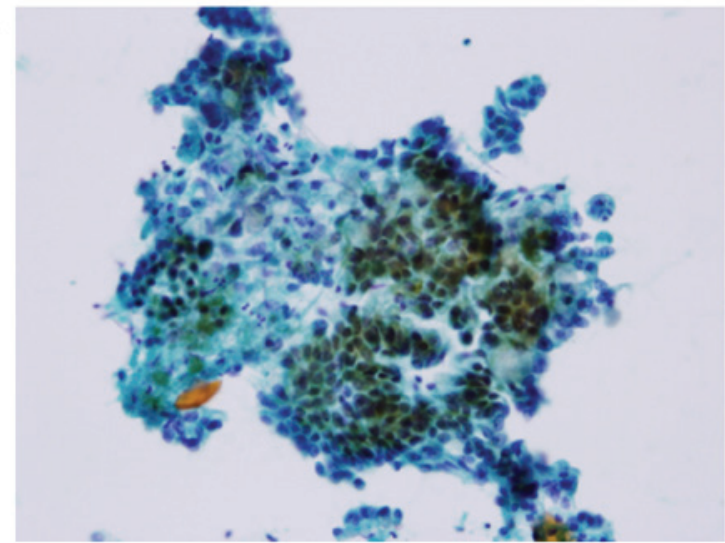

Figure 2. Specimens obtained during endoscopic retrograde cholangiopancreatography with (A) Giemsa stain and (B) Papanicolaou stain

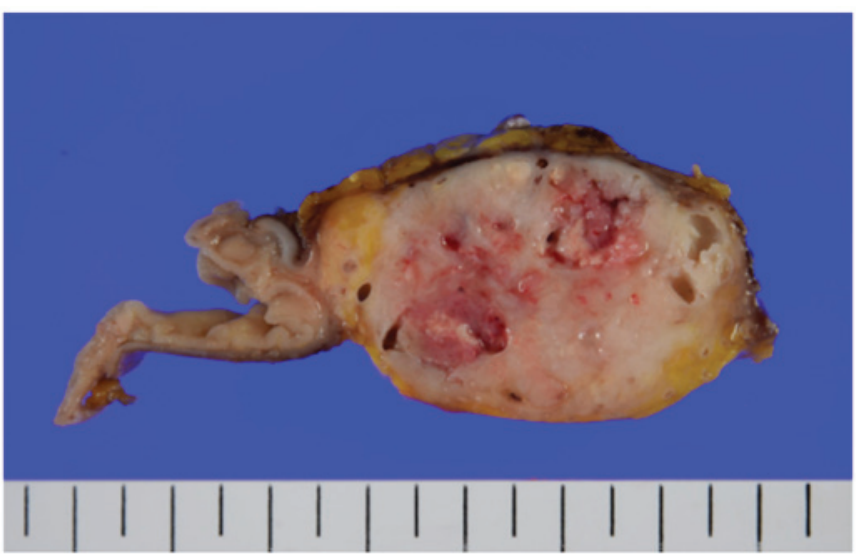

Figure 3. On gross examination, the tumor was located in the head of the pancreas and included mixed cystic and solid components.

trypsin, while the ductal carcinoma component was positive for MUC1, MUC5AC and MUC6. Synaptophysin and chromogranin A were both negative, ruling out a neuroendocrine origin. Based on the aforementioned findings, the patient was diagnosed with mixed ductal-acinar cell carcinoma. Adenocarcinoma cells (of ductal origin) were confirmed on preoperative pancreatic juice cytology. In retrospect, however, small oval-shaped cells with a high N/C ratio were also present; the difference between these cells and normal columnar epithelial cells suggested an acinar cell origin. The ability to distinguish the disease characteristics in the present case, i.e., the ability to conduct special staining methods using cytology specimens, may have facilitated the preoperative diagnosis of this mixed neoplasm.

The mechanism underlying the onset of this mixed neoplasm remains largely elusive; however, a previous study revealed that all pancreatic cells differentiate from developmentally common progenitors (11), namely the pancreatic duodenal homeobox gene-1-positive progenitor cells; however, the ductal lineage diverges at an early stage from p48-positive exocrine and endocrine progenitor cells $(12,13)$. Pancreatic endocrine cells originate from embryonic Ngn-3-expressing cells (14). Also, under certain conditions, acinar cells can transdifferentiate into endocrine cells $(15,16)$. This may be one reason that ductal differentiation is rarer, despite the fact that acinar cell neoplasms often differentiate into pancreatic endocrine cells. Approximately $30 \%$ of all ACC cases exhibit chromogranin and synaptophysin neuroendocrine markers and neuroendocrine tumors may also focally express acinar markers $(17,18)$. 
Table I. The 21 previously reported cases of mixed duct-acinar carcinoma in the English and Japanese literature.

\begin{tabular}{|c|c|c|c|c|c|c|c|c|c|}
\hline Author & Sex & $\begin{array}{c}\text { Age } \\
\text { (years) }\end{array}$ & Chief complaint & Site & $\begin{array}{l}\text { Tumor } \\
\text { diameter }\end{array}$ & Treatment & Follow-up & Prognosis & Refs. \\
\hline Stelow et al & M & 74 & Painless jaundice & Head & 31 & PD & 20 & Alive & (5) \\
\hline Stelow et al & M & 75 & Weight loss, diarrhea & Head & 25 & PD & 39 & Deceased & (5) \\
\hline Stelow et al & M & 73 & Not available & Tail & 20 & & 52 & Deceased & (5) \\
\hline Stelow et al & M & 74 & Weight loss, diarrhea & Head & 40 & PD & 51 & Deceased & (5) \\
\hline Stelow et al & M & 70 & Pain & Head & 40 & PD & 38 & Deceased & (5) \\
\hline Stelow et al & $\mathrm{F}$ & 77 & Weight loss & Head & 30 & $\mathrm{PD}$ & 9 & Deceased & (5) \\
\hline Stelow et al & M & 77 & Weight loss, pain & Head & 37 & $\operatorname{Rdx}$ & 0.5 & Deceased & (5) \\
\hline Stelow et al & $\mathrm{M}$ & 52 & Pain & Head & 55 & $\mathrm{PD}$ & 12 & Deceased & (5) \\
\hline Stelow et al & M & 76 & Painless jaundice & Head & 35 & PD & 8 & Deceased & (5) \\
\hline Stelow et al & $\mathrm{M}$ & 79 & Painless jaundice & Head & 34 & PD & 11 & Alive & (5) \\
\hline Stelow et al & M & 69 & Painless jaundice & Head & 54 & $\mathrm{PD}$ & 36 & Alive & (5) \\
\hline Webb & M & 71 & Not mentioned & Head & 30 & - & 12 & Deceased & (6) \\
\hline Webb & $\mathrm{F}$ & 51 & Not mentioned & Tail & - & - & 3 & Deceased & (6) \\
\hline Webb & $\mathrm{F}$ & 51 & Not mentioned & Tail & 30 & - & 4 & Deceased & (6) \\
\hline Webb & M & 85 & Not mentioned & Tail & - & - & 6 & Deceased & (6) \\
\hline Sakata et al & $\mathrm{F}$ & 67 & Abdominal pain & Body-tail & 110 & $\mathrm{DP}$ & 7 & Deceased & (7) \\
\hline Inaba et al & M & 63 & Abdominal pain & Head & 65 & $\mathrm{PD}+\mathrm{HR}$ & 39 & Alive & (8) \\
\hline Goto et al & $\mathrm{F}$ & 75 & Weight loss & Head-body & 43 & TP & 6 & Alive & (9) \\
\hline Sakai et al & M & 63 & Worsening of diabetes & Head & 35 & PD & 8 & Deceased & (10) \\
\hline Shonaka et al & $\mathrm{F}$ & 71 & Epigastric discomfort & Head/tail & $35 / 72$ & $\mathrm{TP}$ & 36 & Alive & (4) \\
\hline Present case & M & 74 & Hyperglycemia & Head & 35 & $\mathrm{PD}$ & 12 & Deceased & \\
\hline
\end{tabular}

DP, distal pancreatectomy; PD, pancreaticoduodenectomy; HR, hepatic resection; TP, total pancreatectomy; Rdx, radiation therapy; M, male; F, female.
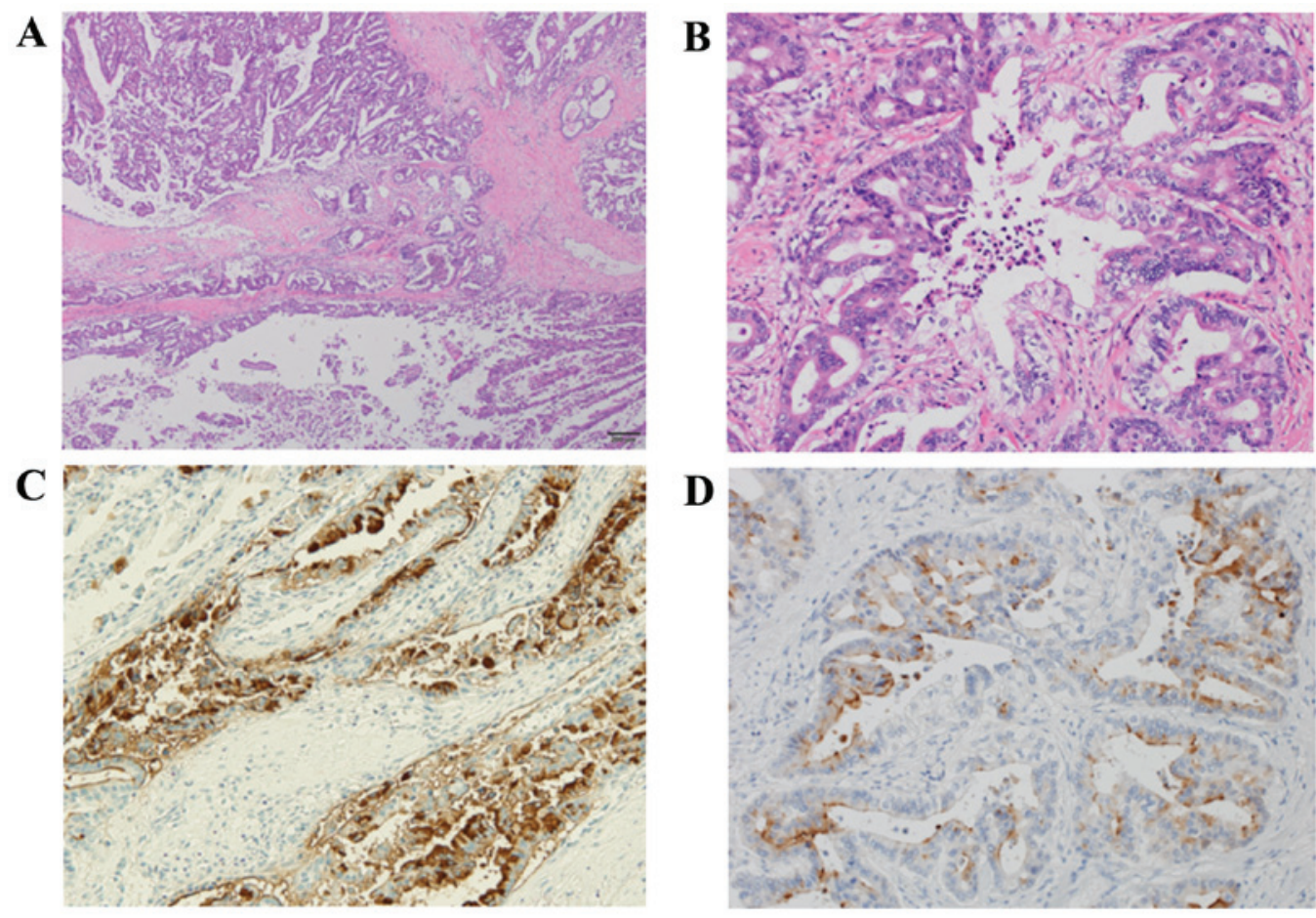

Figure 4. Histopathological findings. Hematoxylin and eosin staining at a magnification of (A) x40 and (B) x200; (C) CA19-9 staining, magnification, x100; (D) trypsin staining, magnification, x200). (A) The lesion was primarily located in the main pancreatic duct; clear differentiation and partial invasion into the pancreatic parenchyma were observed. (B) The two components of the tumor may be seen. The immunohistological examination demonstrated (C) positivity for epithelial tumor markers and carbohydrate antigen 19-9 and (D) a component that was diffusely positive for trypsin. 
In terms of molecular pathology, pancreatic ductal carcinoma progresses through a multistage process from a premalignant lesion to invasive carcinoma due to the accumulation of mutations in KRAS and other genes; however, acinar cell carcinoma almost never harbors $K R A S$ mutations or mutations of tumor suppresor genes such as P16, DPC4/Smad4, or P53 (19-21). As described above, the mechanism underlying the mixture of biologically different neoplasms is unclear and requires further investigation.

Mixed neoplasms of the pancreas are extremely rare, with only a few reports published in the literature to date. The developmental and clinical characteristics of these tumors have not been fully elucidated. Owing to the small number of reports on mixed pancreatic carcinoma, there is no consistent trend regarding outcome. We hope that further accumulation of cases will lead to the elucidation of the mechanism of onset and the establishment of optimal multimodal treatment in the future.

\section{Acknowledgements}

The authors would like to thank Sugako Kajiwara and Jun Uchida for their technical assistance.

\section{Funding}

No funding was received.

\section{Availability of data and materials}

Not applicable.

\section{Authors' contributions}

TS designed the current study and wrote the manuscript. TH and HK analyzed and interpreted the data and wrote the manuscript. YN, YA, FF, RM, TO, IS, AH and TT collected and interpreted the data and critically revised the manuscript. All authors approved the final version of the manuscript, and agree to be accountable for all aspects of the work.

\section{Ethics approval and consent to participate}

Not applicable.

\section{Patient consent for publication}

Not applicable.

\section{Competing interests}

The authors declare that they have no competing interests to disclose.

\section{References}

1. Ohike N, Jürgensen A, Pipeleers-Marichal M and Klöppel G: Mixed ductal-endocrine carcinomas of the pancreas and ductal adenocarcinomas with scattered endocrine cells: Characterization of the endocrine cells. Virchows Arch 442: 258-265, 2003.
2. Hruban RH, Pitman MB and Klimstra DS: Tumors of the Pancreas, AFIP Atlas of Tumor Pathology. Fourth series, Fascicle 6. American Registry of Pathology, Washington, DC, pp191-218, 2007.

3. Bosman FT, Carneiro F, Hruban RH and Theise ND: WHO Classification of Tumours of the Digestive System. 9th edition. IARC/WHO, Lyon, pp279-337, 2010.

4. Shonaka T, Inagaki M, Akabane H, Yanagida N, Shomura H, Yanagawa N, Oikawa K and Nakano S: Total pancreatectomy for metachronous mixed acinar-ductal carcinoma in a remnant pancreas. World J Gastroenterol 20: 11904-11909, 2014.

5. Stelow EB, Shaco-Levy R, Bao F, Garcia J and Klimstra DS: Pancreatic acinar cell carcinomas with prominent ductal differentiation: Mixed acinar ductal carcinoma and mixed acinar endocrine ductal carcinoma. Am J Surg Pathol 34: 510-518, 2010

6. Webb JN: Acinar cell neoplasms of the exocrine pancreas. J Clin Pathol 30: 103-112, 1977.

7. Sakata T, Mimura H, Takakura N, Hamazaki K, Kin H, Kimura T, Hosoba T, Orita K, Mizobuchi K, Taguchi K, et al: A case of mixed duct and acinar cell carcinoma the pancreas showing peculiar modes of spread. J Biliary Tract Pancreas 8: 1349-1353, 1987 (In Japanese).

8. Inaba N, Kasahara K, Kashii A, Kanazawa K, Yamaguchi T, Saito K and Kamisawa T: Mixed ductal and acinar cell cancer of the pancreas head; report of a case. Nihon Geka Gakkai Zasshi 88: 773-778, 1987 (In Japanese).

9. Goto H, Yamazaki Y, Takeuchi T, Kokehara N, Ota M, Ohashi N, Kusakawa M and Soga T: Total pancreatectomy for combined carcinoma of the pancreas-report of a case. J Biliary Tract Pancreas 9: 463-467, 1988 (In Japanese).

10. Sakai M, Takeda S, Ishikawa T, Kanazumi N, Inoue S, Kaneko T, Nakao A and Nagasaka T: Mixed duct-acinar cell carcinoma of the pancreas: Report of a case. Jpn J Gastroenterol Surg 38: 1821-1827, 2005.

11. Fishman MP and Melton DA: Pancreatic lineage analysis using a retroviral vector in embryonic mice demonstrates a common progenitor for endocrine and exocrine cells. Int J Dev Biol 46: 201-207, 2002.

12. Edlund H: Pancreas: How to get there from the gut? Curr Opin Cell Biol 11: 663-668, 1999.

13. Krapp A, Knöfler M, Ledermann B, Bürki K, Berney C, Zoerkler N, Hagenbüchle $\mathrm{O}$ and Wellauer PK: The bHLH protein PTF1-p48 is essential for the formation of the exocrine and the correct spatial organization of the endocrine pancreas. Genes Dev 12: 3752-3763, 1998.

14. Offield MF, Jetton TL, Labosky PA, Ray M, Stein RW, Magnuson MA, Hogan BL and Wright CV: PDX-1 is required for pancreatic outgrowth and differentiation of the rostral duodenum. Development 122: 983-995, 1996.

15. Song KH, Ko SH, Ahn YB, Yoo SJ, Chin HM, Kaneto H, Yoon KH, Cha BY, Lee KW and Son HY: In vitro transdifferentiation of adult pancreatic acinar cells into insulin-expressing cells. Biochem Biophys Res Commun 316: 1094-1100, 2004.

16. Minami K, Okuno M, Miyawaki K, Okumachi A, Ishizaki K, Oyama K, Kawaguchi M, Ishizuka N, Iwanaga T and Seino S: Lineage tracing and characterization of insulin-secreting cells generated from adult pancreatic acinar cells. Proc Natl Acad Sci USA 102: 15116-15121, 2005 .

17. Ohike N and Morohoshi T: Pathological assessment of pancreatic endocrine tumors for metastatic potential and clinical prognosis. Endocr Pathol 16: 33-40, 2005.

18. Ohike N, Kosmahl M and Klöppel G: Mixed acinar-endocrine carcinoma of the pancreas. A clinicopathological study and comparison with acinar-cell carcinoma. Virchows Arch 445: 231-235, 2004

19. Hoorens A, Lemoine NR, McLellan E, Morohoshi T, Kamisawa T, Heitz PU, Stamm B, Rüschoff J, Wiedenmann B and Klöppel G: Pancreatic acinar cell carcinoma. An analysis of cell lineage markers, p53 expression, and Ki-ras mutation. Am J Pathol 143: 685-698, 1993.

20. Moore PS, Orlandini S, Zamboni G, Capelli P, Rigaud G, Falconi M, Bassi C, Lemoine NR and Scarpa A: Pancreatic tumours: Molecular pathways implicated in ductal cancer are involved in ampullary but not in exocrine nonductal or endocrine tumorigenesis. Br J Cancer 84: 253-262, 2001.

21. Terhune PG, Heffess CS and Longnecker DS: Only wild-type c-Ki-ras codons 12,13 , and 61 in human pancreatic acinar cell carcinomas. Mol Carcinog 10: 110-114, 1994. 